\title{
A goal programming procedure for solving fuzzy multiobjective fractional linear programming problems
}

\author{
Tunjo Pericí, ${ }^{1, *}$ Zoran Babić ${ }^{2}$ and Sead Rešić ${ }^{3}$ \\ ${ }^{1}$ Faculty of Economics and Business, University of Zagreb \\ Trg J. F. Kennedyja 6, 10000 Zagreb, Croatia \\ E-mail: 〈tperic@efzg.hr〉 \\ ${ }^{2}$ Faculty of Economics, University of Split \\ Cvite Fiskovića 5, 21000 Split, Croatia \\ E-mail:〈babic@efst.hr〉 \\ ${ }^{3}$ Faculty of Science, University of Tuzla \\ Ulica Dr. Tihomila Markovica br.1, 75000 Tuzla, BiH \\ E-mail: 〈sresic@gmail.com〉
}

\begin{abstract}
This paper presents a modification of Pal, Moitra and Maulik's goal programming procedure for fuzzy multiobjective linear fractional programming problem solving. The proposed modification of the method allows simpler solving of economic multiple objective fractional linear programming (MOFLP) problems, enabling the obtained solutions to express the preferences of the decision maker defined by the objective function weights. The proposed method is tested on the production planning example.
\end{abstract}

Key words: Multiple objective fractional linear programming, linear goal programming, production plan optimization.

Received: October 3, 2014; accepted: December 30, 2014; available online: December 30, 2014

\section{Introduction}

In some economic problems, goals can be more suitably expressed as a ratio of two economic magnitudes. Thus labour productivity is expressed as the ratio of output and the total labour input or as the ratio of output and the total cost invested in it, while profitability is expressed as the ratio of the total income and the total costs in a particular period of time or as the ratio of profit and capital engaged during a particular period. The goals expressed as the ratio of economic magnitudes can reflect the quality of business results better. Also, the

${ }^{*}$ Corresponding author. 
goals expressed in this way enable us to make more adequate comparisons between business entities.

Therefore, if the goals are expressed as the ratio of two economic magnitudes, and if the parameters and variables in the model are linear, then the optimization of economic problems (e.g., optimization of the production plan, optimization of the financial structure, optimization of catering, etc.) requires multiobjective fractional linear programming. The problem of fractional linear programming with one objective function was extensively researched in the second half of the twentieth century and efficient methods were developed for solving such problems ([4], [5], [7], [12]).

However, in MOFLP problems, to determine an efficient (Pareto optimal) solution is technically demanding when goal functions are fractional by linear. Solving multiobjective fractional linear programming models is limited to a small number of multiobjective programming methods which are not efficient enough either from the viewpoint of the analyst or the decision maker ([2], [3], [7], [11], [13], [14], [21]). A special problem arises in the application of goal programming methods as by adding the deviation variables $\mathrm{d}^{-}$and $\mathrm{d}^{+}$nonlinear constraints are obtained which cause numerical problems in model solving. Several methods have been developed that use goal programming to solve multiobjective fractional linear programming models ([9], [10], [16], [17], [18], [19], [22]), but there are few studies in which these methods are applied and tested in solving real economic problems ([6], [14], and [20]).

To solve the multiobjective fractional linear programming model with fuzzy functions Pal et al. [18] proposed the goal function linearization method which enables the application of the goal programming method in such problems. The proposed method was tested in two numeric examples with two variables. When testing the proposed method on the example of production plan optimization with fuzzy fractional linear objective functions we discovered the possibility to improve the method both from the standpoint of the analyst and the standpoint of decision maker. Therefore, this study proposes a modification of Pal et al. [18] goal programming procedure to solve the fuzzy multi objective fractional linear programming. In the proposed modification, the decision maker is only asked to provide the information on the relative importance of goal functions. The proposed method modification is tested on the example of production plan optimization for a particular period. 


\section{Goal programming methodology for solving MOFLP problems}

\subsection{MOFLP model}

A MOFLP model can be written as

$$
\begin{aligned}
& \max Z=\left[z_{1}, z_{2}, \ldots, z_{K}\right] \\
& \text { s. t. } A x^{*} b, \quad x \geq 0,
\end{aligned}
$$

where $z_{k}=\frac{\sum_{j=1}^{n} c_{k j} x_{j}+c_{0}^{k}}{\sum_{j=1}^{n} d_{k j} x_{j}+d_{0}^{k}}, k=1,2, \ldots, K$, are fractional linear functions, $A$ is an $m \mathrm{x} n$ matrix, $x$ is an $n$-dimensional nonnegative variable vector, $b$ is an $m-$ dimensional vector, and $*$ represents symbols $\leq,=$ and $\geq$.

Numerous methods are proposed for solving model (1), which are not efficient enough from the point of view of decision makers and analysts.

\subsection{Pal, Moitra and Maulik's method}

Pal, Moitra and Maulik proposed the following linearization model to solve fuzzy MOFLP problems by goal programming methods:

Find $x$ so as to

$$
\begin{aligned}
\text { Minimize } & z=\sum_{k=1}^{K} w_{k}^{-} D_{k}^{-} \\
\text {and satisfy } & C_{k} x+D_{k}^{-}-D_{k}^{+}=G_{k} \\
\text { subject to } & A x^{*} b \\
\text { and } & -d_{k} x+D_{k}^{-} \leq d_{0}^{k}, \\
& x \geq 0, \\
& D_{k}^{-}, D_{k}^{+} \geq 0,
\end{aligned}
$$

where $z$ represents the fully achievement function consisting of the weighted under-deviational variables, and numerical weights $w_{k}$ represent the relative importance of achieving the aspired levels of the respective fuzzy goals subject to the constraint set in the decision situation;

$C_{k}=L_{k} C_{k}-L_{k}^{\prime} d_{k}, \quad G_{k}=L_{k}^{\prime} d_{0}^{k}-L_{k} C_{0}^{k}, \quad\left(L_{k}=\frac{1}{g_{k}-l_{k}}, L_{k}^{\prime}=1+L_{k} l_{k}\right), \quad w_{k}^{-}=\frac{1}{g_{k}-l_{k}}$,

(for functions to be maximized) or $w_{k}^{-}=\frac{1}{u_{k}-g_{k}}$, (for functions to be minimized), $l_{k}$ is the lowest value of the $k$ th objective function, $u_{k}$ is the 
biggest value of the $k$ th objective function, while $g_{k}$ is the value determined by the decision maker.

\subsection{The modification of the Pal, Moitra and Maulik's method}

To solve the problem of multiobjective fractional linear programming with vaguely defined goals of the decision maker by applying the goal programming method we propose a modification of the method described above. As the decision maker is not able to precisely express the value of goals to be achieved, we propose to determine the decision maker's goal levels by using the pay-off table formed by optimizing each single objective function on the given set of constraints. In the objective functions to be maximized, the upper limit is the maximal value of objective functions obtained by their maximization on the given set of constraints while the bottom limit is the smallest value of the given objective function in the pay-off table. In the objective functions to be minimized, the upper limit is the highest value of the objective function in the pay-off table while the bottom limit is the minimal value of the objective function obtained by its minimization on the given set of constraints.

In the proposed modification, the decision maker is asked to provide only the information on the objective functions weight. If the decision maker is not able to express the relative importance of the objective functions by weights, the weights can be calculated by applying one of the weight determining methods. An interesting idea for calculating objective functions weights is presented in [15].

To solve the multiobjective fractional linear programming model with vaguely expressed goals of the decision maker by applying the goal programming method for objective functions that are to be maximized we propose linearization of the fractional linear functions in the following way:

$$
\begin{gathered}
\frac{\sum_{j=1}^{n} c_{k j} x_{j}+c_{0}^{k}}{\sum_{j=1}^{n} d_{k j} x_{j}+d_{0}^{k}} \geq z_{k}^{*} / \cdot\left(\sum_{j=1}^{n} d_{k j} x_{j}+d_{0}^{k}\right), k=1,2, \ldots, k_{1} \Rightarrow \sum_{j=1}^{n} c_{k j} x_{j}+c_{0}^{k} \geq z_{k}^{*}\left(\sum_{j=1}^{n} d_{k j} x_{j}+d_{0}^{k}\right) \\
\sum_{j=1}^{n}\left(c_{k j}-z_{k}^{*} d_{k j}\right) x_{j} \geq z_{k}^{*} d_{0}^{k}-c_{0}^{k} \Rightarrow \sum_{j=1}^{n} C_{k j} x_{j}+d_{k}^{-}=Z_{k}^{*}, k=1,2, \ldots, k_{1},
\end{gathered}
$$

where $z_{k}^{*}$ is the maximal value of the $k$ th objective function on the given set of constraints, $C_{k j}=c_{k j}-z_{k}^{*} d_{k j}, \quad Z_{k}^{*}=z_{k}^{*} d_{0}^{k}-c_{0}^{k}$, and $d_{k}^{-}$is an under-deviational variable $\left(k=1,2, \ldots, k_{1}\right)$. 
Analogously, way we can linearize the fractional linear functions to be minimized:

$$
\begin{gathered}
\frac{\sum_{j=1}^{n} c_{k j} x_{j}+c_{0}^{k}}{\sum_{j=1}^{n} d_{k j} x_{j}+d_{0}^{k}} \leq z_{k}^{*} / \cdot\left(\sum_{j=1}^{n} d_{k j} x_{j}+d_{0}^{k}\right) \Rightarrow \sum_{j=1}^{n} c_{k j} x_{j}+c_{0}^{k} \leq z_{k}^{*}\left(\sum_{j=1}^{n} d_{k j} x_{j}+d_{0}^{k}\right) \\
\sum_{j=1}^{n}\left(c_{k j}-z_{k}^{*} d_{k j}\right) x_{j} \leq z_{k}^{*} d_{0}^{k}-c_{0}^{k} \Rightarrow \sum_{j=1}^{n} C_{k j} x_{j}-d_{k}^{+}=Z_{k}^{*}, k=k_{1}, k_{1}+1, \ldots, K,
\end{gathered}
$$

where $Z_{k}^{*}$ is the minimal value of the $k$ th objective function on the given set of constraints, and $\boldsymbol{d}_{k}^{+}$is an over-deviational variable $\left(k=k_{1}+1, k_{1}+2, \ldots, K\right)$.

Therefore, the goal programming model is presented as

$$
\begin{array}{ll} 
& \min g_{k}\left(d_{k}^{-}, d_{k}^{+}\right) \\
\text {subject to } & C_{k j} x_{j}+d_{k}^{-}=Z_{k}^{*}, \quad k=1,2, \ldots, k_{1}, \\
& C_{k j} x_{j}-d_{k}^{+}=Z_{k}^{*}, k=k_{1}, k_{1}+1, \ldots, K, \\
& A x^{*} b, \\
& x \geq 0, \\
& D_{k}^{-}, D_{k}^{+} \geq 0 .
\end{array}
$$

To solve model (5) we can use numerous GP approaches for solving MOLGP models:

(1) The min-max form:

Min $\max q_{k}\left(\boldsymbol{d}_{k}^{-}, \boldsymbol{d}_{k}^{+}\right)$

$$
\begin{array}{ll}
\text { s. t. } & \sum_{j=1}^{n} C_{k j} x_{j}+d_{k}^{-}=Z_{k}^{*}, k=1,2, \ldots, k_{1}, \\
& \sum_{j=1}^{n} C_{k j} x_{j}-d_{k}^{+}=Z_{k}^{*}, k=k_{1}, k_{1}+1, \ldots, K, \\
& A x^{*} b, \\
& x \geq 0, d_{k}^{-}, d_{k}^{+} \geq 0 .
\end{array}
$$

Therefore, $d_{k}^{-}$appears in the constraint formed by the objective function to be maximized, while $d_{k}^{+}$appears in the constraint formed by the objective function to be minimized. 
To solve model (6), according to papers [23] and [24], we can form the following specific linear programming model, which can be solved by the simplex method:

$$
\begin{array}{ll}
\text { Min } & \lambda \\
\text { s. t. } & \lambda-d_{k}^{-} \geq 0, k=1,2, \ldots, k_{1}, \\
& \lambda-d_{k}^{+} \geq 0, k=k_{1}+1, k_{1}+2, \ldots, K, \\
& \sum_{j=1}^{n} C_{k j} x_{j}+d_{k}^{-}=Z_{k}^{*}, \quad k=1,2, \ldots, k_{1}, \\
& \sum_{j=1}^{n} C_{k j} x_{j}-d_{k}^{+}=Z_{k}^{*}, \quad k=k_{1}, k_{1}+1, \ldots, K, \\
& A x^{*} b, \\
& x \geq 0, \lambda, d_{k}^{-}, d_{k}^{+} \geq 0 .
\end{array}
$$

(2) The minimization of the sum of deviations form:

$$
\begin{array}{ll}
\text { Min } & \sum_{k=1}^{K} g_{k}\left(d_{k}^{-}, d_{k}^{+}\right) \\
\text {s. t. } & \sum_{j=1}^{n} C_{k j} x_{j}+d_{k}^{-}=Z_{k}^{*}, \quad k=1,2, \ldots, k_{1}, \\
& \sum_{j=1}^{n} C_{k j} x_{j}-d_{k}^{+}=Z_{k}^{*}, \quad k=k_{1}, k_{1}+1, \ldots, K, \\
& A x^{*} b, \\
& x \geq 0, d_{k}^{-}, d_{k}^{+} \geq 0 .
\end{array}
$$

Model (8) is a linear programming model which can be solved by using the simplex method.

(3) The min-max weighting form:

$$
\begin{array}{ll}
\text { Min } & g_{k}\left(w_{k} d_{k}^{-}, w_{k} d_{k}^{+}\right) \\
\text {s.t. } & \sum_{j=1}^{n} C_{k j} x_{j}+d_{k}^{-}=Z_{k}^{*}, k=1,2, \ldots, k_{1}, \\
& \sum_{j=1}^{n} C_{k j} x_{j}-d_{k}^{+}=Z_{k}^{*}, \quad k=k_{1}, k_{1}+1, \ldots, K, \\
& A x^{*} b, \\
& x \geq 0, d_{k}^{-}, d_{k}^{+} \geq 0 .
\end{array}
$$

where $\mathrm{w}_{k}, \sum_{k=1}^{K} w_{k}=1,(k=1,2, \ldots, K)$ are weights determined by the decision maker.

To solve model (9), we can form the following specific linear programming model to be solved by the simplex method ([24], [25]): 


$$
\begin{array}{ll}
\text { Min } & \lambda \\
\text { s.t. } & \lambda-w_{k} d_{k}^{-} \geq 0, k=1,2, \ldots, k_{1}, \\
& \lambda-w_{k} d_{k}^{+} \geq 0, k=k_{1}+1, k_{1}+2, \ldots, K, \\
& \sum_{j=1}^{n} C_{k j} x_{j}+d_{k}^{-}=Z_{k}^{*}, \quad k=1,2, \ldots, k_{1}, \\
& \sum_{j=1}^{n} C_{k j} x_{j}-d_{k}^{+}=Z_{k}^{*}, \quad k=k_{1}, k_{1}+1, \ldots, K, \\
& A x^{*} b, \\
& x \geq 0, \lambda, d_{k}^{-}, d_{k}^{+} \geq 0 .
\end{array}
$$

(iv) The preemptive priority form: In this form, the $K$ objectives are rearranged according to decision maker's priority levels, the highest priority goal is considered first, then the second and so on. The general lexicographical goal program is:

$$
\begin{aligned}
\min a=\min _{k \in P_{i}} g_{k}( & \left.d_{k}^{-}, d_{k}^{+}\right): i=1,2, \ldots, I ; k=1,2, \ldots, K \\
\text { s. t. } \quad & \sum_{j=1}^{n} C_{k j} x_{j}+d_{k}^{-}=Z_{k}^{*}, k=1,2, \ldots, k_{1}, \\
& \sum_{j=1}^{n} C_{k j} x_{j}-d_{k}^{+}=Z_{k}^{*}, k=k_{1}, k_{1}+1, \ldots, K, \\
& A x^{*} b, \\
& x \geq 0, d_{k}^{-}, d_{k}^{+} \geq 0,
\end{aligned}
$$

where $I$ is the number of priority levels and $k \in P_{i}$ means that the $k$ th goal is in the $i$ th priority level.

Model (11) is a linear programming model which can be solved by the simplex method.

According to [8] and [22], the solutions obtained by above models are efficient (Pareto optimal).

\section{Practical application: production plan optimization}

\subsection{The problem}

The data are taken from paper [19]. In the period from January to December of the following year, the company plans to manufacture thirty different products that will be denoted by $x_{1}$ to $x_{30}$. The data taken from the tables of paper [19] are represented in the following way: the gross profit per product $\left(c_{1 j}\right)$, the output $\left(c_{2 j}\right)$, the gross profit from export per product $\left(c_{3 j}\right)$, variable costs per 
product $\left(d_{1 j}, d_{3 j}\right)$ and the total labour invested by the shop floor workers $\left(d_{2 j}\right)$, the expected gross profits from other company activities in the given period $\left(c_{0}^{1}\right)$, the expected total fixed costs in the given period $\left(d_{0}^{1}, d_{0}^{3}\right)$, and the expected total labour invested by other company employees $\left(d_{0}^{2}\right)$.

\subsection{The MOFLP model}

The company wants to optimize its production plan for the given period maximizing the following three objective functions:

(1) profitability measured by the ratio of the total expected gross profits and the total expected costs,

(2) productivity measured by the ratio of the expected total output and the expected total labour invested, and

(3) export profitability measured by the ratio of the expected gross profits from exports and the expected total costs.

The MOFLP model looks like this:

a) Objective functions

Profitability

Productivity

$$
\max z_{1}=\frac{\sum_{j=1}^{30} c_{1 j} x_{j}+c_{0}^{1}}{\sum_{j=1}^{30} d_{1 j} x_{j}+d_{0}^{1}},
$$

Export profitability

$$
\max z_{2}=\frac{\sum_{j=1}^{30} c_{2 j} x_{j}}{\sum_{j=1}^{30} d_{2 j} x_{j}+d_{0}^{2}},
$$

$$
\max z_{3}=\frac{\sum_{j=1}^{30} c_{3 j} X_{j}}{\sum_{j=1}^{30} d_{3 j} x_{j}+d_{0}^{3}} .
$$


b) Constraints

$$
\begin{aligned}
& \text { s.t. } \sum_{j=1}^{30} a_{1 j} x_{j} \leq b_{1}, \quad \sum_{j=1}^{30} a_{2 j} x_{j} \leq b_{2}, \sum_{j=1}^{30} a_{3 j} x_{j} \leq b_{3}, \sum_{j=1}^{30} a_{4 j} x_{j} \leq b_{4}, \sum_{j=1}^{30} a_{5 j} x_{j} \leq b_{5}, \\
& \sum_{j=1}^{30} a_{6 j} x_{j} \leq b_{6}, x_{j} \leq 500000, j=1,2,3,6,7,9,10,11,12,13,14,16,17,18, \\
& 19,20,21,22,23,24,25,30, x_{4} \leq 345000, \quad x_{5} \leq=575000, x_{8} \leq 172500, \\
& x_{15} \leq 230000, x_{2} \leq 345000, x_{27} \leq 230000, x_{28} \leq 300000, x_{29} \leq 264500, \\
& x_{6} \geq 115000, x_{13} \geq 172500, x_{16} \geq 115000, x_{j} \geq 0(j=1,2, \ldots, 30) .
\end{aligned}
$$

\subsection{Model solving}

Model (12)-(15) is first solved by the Charnes - Cooper method maximizing each of the objective functions on the given set of constraints using Lindo 6.1. The obtained marginal solutions are presented in Table 1.

\begin{tabular}{|c|c|c|c|}
\hline $\begin{array}{c}\text { Objective } \\
\text { function }\end{array}$ & $z_{1}$ & $z_{2}$ & $z_{3}$ \\
\hline $\max z_{1}$ & $0.273726\left(z_{1}^{*}\right)$ & 2.461424 & 0.067538 \\
\hline $\max z_{2}$ & 0.096546 & $5.563876\left(z_{2}^{*}\right)$ & 0.07027 \\
\hline $\max z_{3}$ & 0.057198 & 3.162149 & $0.256522\left(z_{3}^{*}\right)$ \\
\hline
\end{tabular}

Table 1. Model (12)-(15) marginal solutions

It is obvious from the above table that the objective functions are conflicting, which makes it difficult for the decision maker to select the preferred solution from the marginal solutions. Therefore, one or several compromise solutions have to be proposed to the decision maker and these solutions also have to be efficient (Pareto optimal).

The decision maker is asked to provide the information on the relative importance of objective functions. If the decision maker cannot provide such information, the analyst can use the data from the table of payments to calculate the weights of objective functions and suggest the decision maker to accept the solution obtained in that way, or they can calculate the set of efficient solutions obtained by varying the objective functions weights. Here we will present the solutions obtained by applying the proposed methodology for linearization of objective functions by using of the above stated four approaches for solving goal programming models. By applying model (3) to the above presented data, the objective functions are linearized in the following way: 


$$
\begin{aligned}
& \frac{\sum_{j=1}^{30} c_{k j} x_{j}+c_{0}^{k}}{\sum_{j=1}^{30} d_{k j} x_{j}+d_{0}^{k}} \leq z_{k}^{*} / \cdot\left(\sum_{j=1}^{30} d_{k j} x_{j}+d_{0}^{k}\right), k=1,2,3 \sum_{j=1}^{30} c_{k j} x_{j}+c_{0}^{k} \leq z_{k}^{*}\left(\sum_{j=1}^{30} d_{k j} x_{j}+d_{0}^{k}\right) \Rightarrow \\
& \sum_{j=1}^{30} c_{k j} x_{j}-z_{k}^{*} \sum_{j=1}^{30} d_{k j} x_{j} \leq z_{k}^{*} d_{0}^{k}-c_{0}^{k} \Rightarrow \sum_{j=1}^{30} c_{k j} x_{j}-z_{k}^{*} \sum_{j=1}^{30} d_{k j} x_{j}+d_{k}^{-}=z_{k}^{*} d_{0}^{k}-c_{0}^{k} \cdot
\end{aligned}
$$

The goal programming models look like this:

(1) The min-max form

$$
\begin{array}{ll}
\min & g\left(d_{k}^{-}\right), k=1,2,3 \\
\text { s. t. } & \sum_{j=1}^{30} c_{k j} x_{j}-z_{k}^{*} \sum_{j=1}^{30} d_{k j} x_{j}+d_{k}^{-}=z_{k}^{*} d_{0}^{k}-c_{0}^{k}
\end{array}
$$

constraints (15).

Model (17) is solved by the Zimmermann approach to solving linear programming models:

$$
\begin{array}{ll}
\min & \lambda \\
\mathrm{s.t.} & \lambda-d_{1}^{+} \geq 0 \\
& \lambda-d_{2}^{-} \geq 0 \\
& \lambda-d_{3}^{-} \geq 0 \\
& \sum_{j=1}^{30} c_{k j} x_{j}-z_{k}^{*} \sum_{j=1}^{30} d_{k j} x_{j}+d_{k}^{-}=z_{k}^{*} d_{0}^{k}-c_{0}^{k}
\end{array}
$$

constraints (15).

(2) The minimization of the sum of deviations form:

$$
\begin{array}{ll}
\min & \sum_{k=1}^{3} d_{k}^{-} \\
\text {s. t. } & \sum_{j=1}^{30} c_{k j} x_{j}-z_{k}^{*} \sum_{j=1}^{30} d_{k j} x_{j}+d_{k}^{-}=z_{k}^{*} d_{0}^{k}-c_{0}^{k}
\end{array}
$$

constraints (15).

(3) The minimization of the weighted sum of deviations form:

$$
\begin{array}{ll}
\min & \sum_{k=1}^{3} w_{k} d_{k}^{-} \\
\text {s. t. } & \sum_{j=1}^{30} c_{k j} x_{j}-z_{k}^{*} \sum_{j=1}^{30} d_{k j} x_{j}+d_{k}^{-}=z_{k}^{*} d_{0}^{k}-c_{0}^{k}
\end{array}
$$

constraints (15),

where $w_{1}=0.5, w_{2}=0.3, w_{3}=0.2$ are weights of objective functions determined by the decision maker.

Model (20) has been solved by solving the following linear programming model: 


$$
\begin{array}{ll}
\min & \lambda \\
\text { s. t. } & \sum_{j=1}^{30} c_{k j} x_{j}-z_{k}^{*} \sum_{j=1}^{30} d_{k j} x_{j}+d_{k}^{-}=z_{k}^{*} d_{0}^{k}-c_{0}^{k}
\end{array}
$$

constraints (15)

$$
\begin{aligned}
& \lambda-w_{1} d_{1}^{-} \geq 0, \\
& \lambda-w_{2} d_{2}^{-} \geq 0, \\
& \lambda-w_{3} d_{3}^{-} \geq 0,
\end{aligned}
$$

where $\lambda, w_{1}, w_{2}, w_{3}, d_{1}^{-}, d_{2}^{-}, d_{3}^{-} \geq 0$, and $\sum_{k=1}^{3} w_{k}=1$.

(4) The preemptive priority form: The three objectives are ranked according to their priority: goal 1 has priority 1 , goal 2 has priority 2 , and goal 3 has priority 3. The general lexicographical goal program is:

$$
\begin{gathered}
\min \mathrm{a}=\min _{k \in P_{i}} g_{k}\left(d_{k}^{-}\right): i=1,2,3 ; k=1,2,3 \\
\text { s. t. } \sum_{j=1}^{30} c_{k j} x_{j}-z_{k}^{*} \sum_{j=1}^{30} d_{k j} x_{j}+d_{k}^{-}=z_{k}^{*} d_{0}^{k}-c_{0}^{k}, k=1,2,3 \\
\text { constraints (15), }
\end{gathered}
$$

where $k \in P_{i}$ means that the $k$ th goal is in the $i$ th priority level. In our model, goal $z_{1}$ is on the first level, goal $z_{2}$ is on the second level, and goal $z_{3}$ is on the third level. Model (22) is solved by using the sequential simplex method.

\subsection{The solutions}

The solutions of models (18), (19), (21) and (22) are shown in Table 2.

\begin{tabular}{|c|c|c|c|}
\hline GP solution & $z_{1}$ & $z_{2}$ & $z_{3}$ \\
\hline$(1)$ & 0.24188 & 3.74423 & 0.224984 \\
\hline$(2)$ & 0.218005 & 4.50649 & 0.256523 \\
\hline$(3)$ & 0.252394 & 3.453361 & 0.203193 \\
\hline$(4)$ & 0.262165 & 3.742245 & 0.155269 \\
\hline
\end{tabular}

Table 2. Goal programming solutions 
The application of the proposed model to solve a financial programming optimization problem gives four different solutions. Obtaining the solutions to models (18) and (19) does not require additional information from the decision maker. In this case, the decision maker must decide which solution to choose. However, if the decision maker can give information on the relative importance of the objective functions, they can use solution of model (21) or model (22). In the last two cases, the obtained solutions express decision maker's preferences. Solving model 21 enables the analyst to form a set of nondominated solutions by varying objective functions weights, from which the decision maker can choose the preferred solution.

Which goal programming method should be used depends on the existence of decision maker's preferences. If the decision maker can express his/her objective function preferences, then it is acceptably to use model (21) or model (22) to solve the problem. Otherwise, the decision maker should use model (18) or model (19).

Comparing the solution presented in Table 2 with the solution obtained by the multiobjective linear programming STEM method [1], we can conclude that the solutions obtained by the goal programming method give more information about the quality of a business enterprise than the solution obtained by the STEM method. The choice of methods depends on the choice of objective functions (linear or fractional) by the decision maker.

\section{Conclusion}

Linearization of fractional linear functions for solving fuzzy multiple objective fractional linear programming problems by using goal programming methodology is proposed in this paper.

The proposed methodology has been tested on the example of production plan optimization in a company which produces textile products for a certain period. The obtained results show the possibility of efficient application of the proposed methodology to the given problem.

Four different goal programming approaches for solving the production plan optimization problem are presented in the paper. The proposed methodology allows using of the lexicographic simplex method in the process of obtaining the preferred solution with the active participation of decision makers in the process.

There are many advantages of the proposed methodology compared to the existing methods.

So the proposed methodology is simple for both the analyst and the decision maker (any additional information from the decision maker is not required). The decision maker can determine objective function weights, and 
thereby the obtained solutions reflect the decision maker's preferences. This method allows the analyst to form a set of efficient solutions by varying the objective function weights, from which the decision maker can choose the preferred solution.

However, the presented approach is limited to solving multi objective fractional linear programming problems with fuzzy goals. Here it is assumed that the decision makers cannot express the desired value of the objective functions.

The main contribution of the paper is the proposed goal programming approach for solving multiobjective fractional linear programming models, which seems to be applicable to solving practical economic problems.

In future research we propose developing a goal programming model which could be applied to solving all kinds of multiobjective fractional linear programming models, not only the models with fuzzy objective functions.

\section{Acknowledgement}

This work has been fully supported by Croatian Science Foundation under the project STRENGTHS No. 9402.

\section{References}

[1] Benayoun, J., de Montgolfier, Tergny, J. and Laritchev, O. (1971). Linear programming with multiple objective functions: Step method (stem), Mathematical Programming 1, 366-375.

[2] Caballero, R. and Hernandez, M. (2006). Restoration of efficiency in a goal programming problem with linear fractional criteria, European Journal of Operational Research, 172, 31-39.

[3] Chakraborty, M. and Gupta, S. (2002) Fuzzy mathematical programming for multi objective linear fractional programming problem, Fuzzy Sets and Systems, 125, 335342.

[4] Charnes, A. and Cooper, W.W. (1961). Management Models of Industrial Applications of Linear Programme (Appendix B), Vol. 1, Wiley, New York.

[5] Craven, B.D. (1988). Fractional Programming, Heldermann Verlag, Berlin.

[6] Gomez, T., Hernandez, M., Leon, M.A, and Caballero, R. (2006). A forest planning problem solved via a linear fractional goal programming model, Forest Ecology and Management, 227, 79-88.

[7] Hannan, E.L. (1981). Linear programming with multiple fuzzy goals, Fuzzy sets and systems, 6, 235-248.

[8] Ignizio, J.P. (1976). Goal Programming and Extensions, Lexington D.C.Health. MA.

[9] Kao, C. and Liu, S.T. (2001). Fractional programming approach to fuzzy weighted average, Fuzzy Sets and Systems, 120, 435-444. 
[10] Kornbluth, J.S.H. and Steuer, R.E. (1981). Goal programming with linear fractional criteria, European Journal of Operational Research, 8, 58-65.

[11] Kornbluth, J.S.H. and Steuer, R.E. (1981). Multiple objective linear fractional programming, Management Science, 27, 1024-1039.

[12] Mehrjerdi, Y.Z. (2011). Solving fractional programming problem through fuzzy goal setting and approximation, Applied Soft Computing, 11, 1735-1742.

[13] Metev, B. and Gueorguieva, D. (2000). A simple method for obtaining weakly efficient points in multiobjective linear fractional programming problems, European Journal of Operational Research, 126, 386-390.

[14] Mishra, B. and Singh, S.R. (2013). Linear fractional programming procedure for multi objective linear programming problem in agricultural system, International Journal of Computer Applications, 60, 45-52.

[15] Mohamed, R.H. (1997). The relationship between goal programming and fuzzy programming, Fuzzy Sets and Systems, 89, 215-222.

[16] Ohta, H. and Yamaguchi, T. (1996). Linear fractional goal programming in consideration of fuzzy solution, European Journal of Operational Research 92, 157165.

[17] Pal, B.B. and Basu, I. (1995). A goal programming method for solving fractional programming problems via dynamic programming, Optimization, 35, 145-157.

[18] Pal, B.B., Moitra, B.N., Maulik, U. (2003) A goal programming procedure for fuzzy multiobjective linear fractional programming problem, Fuzzy Sets and Systems, 139, 395-405.

[19] Perić, T. and Babić, Z.(2009). Determining optimal production program with fuzzy multiple criteria programming method, Proceedingse of the International multiconference of engineers and computer scientists, Hong Kong, 2006-2013.

[20] Perić, T. and Babić, Z. (2012) Financial structure optimization by using a goal programming approach, Croatian Operational Research Review, 3, 150-162.

[21] Sakawa, M. and Yumine, T. (1983). Interactive fuzzy decision making for multiobjective linear fractional programming problems, Large Scale Systems, 5, 105114.

[22] Tiwary, R.N., Dharmar, S. and Rao, J.R. (1987). Fuzzy goal programming - an additive model, Fuzzy Sets and Systems, 24, 27-34.

[23] Zeleny, M., The pros and cons of goal programming, (1981). Computers and Operational Research, 8, 357-359.

[24] Zimmermann, H.-J. (1978). Fuzzy programming and linear programming with severel objective functions, Fuzzy Sets and Systems, 1, 45-55.

[25] Zimmermann, H.-J. (1987). Fuzzy Sets, Decision Making and Expert Systems, Cluwer Academic Publishers, Boston, Dordrecht. 